\title{
AN IMPROVED DECISION SUPPORT SYSTEM BASED ON THE BDM (BIT DECISION MAKING) METHOD FOR SUPPLIER SELECTION USING BOOLEAN AlgEBRA
}

\author{
Gabriel Almazán ${ }^{1}$ \\ ${ }^{1}$ IndustrialEngineering Educational Program, Superior School of Tepeji, Basic Sciences \\ Institute, Autonomous University of Hidalgo, México
}

\begin{abstract}
Based on the BDM (Bit Decision Making) method, the present work presents two contributions: first, the illustration of the use of the technique known as SOP (Sum Of Products) in order to systematize the process to obtain the correlation function for sub-system's mathematical modelling, and second,the provision of capacity to manage a greater than binary but a finite - discrete set of possible subjective qualifications of suppliers at any criterion.
\end{abstract}

\section{KEYWORDS}

Supplier Selection, Decision Support Systems, BDM (Bit Decision Making), Multi Criteria Decision Analysis, Boolean Algebra.

\section{INTRODUCTION}

The present work focuses on providing a contribution to the $\mathrm{BDM}$ (Bit Decision Making) method reported on [1]. In order to accomplish this, we give some basic conceptual and theoretical references that will provide a full operative mechanism to obtain the correlation function that relates the logic binary values assigned to suppliers by the decision maker(s) (inputs) with each sub-system output decision. As a consequence of this, an additional benefit will be the provision of capacity to qualify suppliers in more than just two ways ("yes" or "no"), but in a finitediscrete set of values.

In section 2 we give a brief exposition of BDM method and identify the opportunity areas in the initial proposal, where we visualize our contributions. In section 3 we present the conceptual and theoretical framework that will enable the BDM method to manage a discrete set of values for supplier qualifications. In section 4, we illustrate the application of the conceptual and theoretical background to the case presented at [1]. Finally, at section 5 we propose a variation on a specific sub-system to visualize implications and the benefit of our proposal.

\section{THE BDM METHOD}

\subsection{A brief description}

In BDM method, the decision will be reached considering every supplier's performance on subsystems of the form shown in Table 1. 
Table 1. Truth Table structure for any sub-system.

\begin{tabular}{|l|l|l|l|l|l|}
\hline $\begin{array}{l}\text { Decision Criteria } \\
\text { Pattern Number }\end{array}$ & $\begin{array}{c}\text { First } \\
\text { Criterion }\end{array}$ & $\begin{array}{c}\text { Second } \\
\text { Criterion }\end{array}$ & $\cdots$ & $\begin{array}{c}\text { Nth } \\
\text { Criterion }\end{array}$ & Output \\
\hline 1 & yes/no & yes/no & yes/no & yes/no & Yes/No \\
\hline 2 & yes/no & yes/no & yes/no & yes/no & Yes/No \\
\hline$\ldots$ & yes/no & yes/no & yes/no & yes/no & Yes/No \\
\hline Mth Pattern & yes/no & yes/no & yes/no & yes/no & Yes/No \\
\hline
\end{tabular}

The decision maker will configure the truth table by choosing the criteria combinations that will output a "Yes" or "No" result. Therefore, each sub-system acts as a filter for suppliers. If the output is "Yes", the supplier can continue to the next sub-system, otherwise it will be disqualified,and so on until reaching the last sub-system.

\subsection{Opportunity Areas Identification}

As can be seen in the reference paper [1], the way in which the logical function (that correlates inputs and output in each sub-system) is generated, is not specified. On the other hand, the original method is not able to manage a ranking scheme in supplier'sperformance qualifications at any criterion; for instance, the following degrees:excellent, good, average, poor.

\section{CONCEPTUAL AND THEORETICAL REFERENCES 3.1. Number of possible patterns}

According to combinatorics, the total number of patters for a truth table of $\mathrm{N}$ binary variables will be $2^{\mathrm{N}}$.

\subsection{The SOP (Sum Of Products) Technique}

To obtain a logical function that correlates binary inputs with a binary output, there are two options: in a Sum Of Products (SOP) or in Product Of Sums (POS) form. The Sum of Products (SOP) form will contain a list of terms in which all variables are ANDed (products), then ORed (summed) together.To convert a truth table to a SOP expression, only the rows whose output is "Yes" will produce a term. This AND term should produce a "Yes" with the values of variables in that row; hence, all the variables with value "no" should be logically complemented (inverted).

\subsection{The minimal form}

Boolean algebra axioms and theorems or Karnaugh maps (a graphical tool) could be used in order to find the minimal expression of the logical function that correlates inputs and the output. Basically, this tools help to identify which variables within a term -considerated jointly with other terms, become irrelevant.

\subsection{Binary Coding}

A set of things (characters, symbols, values, etc.) can be represented with a group of bits.Being $x$ the number of objects, the number of bits required $(n)$ can be calculated with:

$$
n=\frac{\log x}{\log 2}
$$




\section{Illustrative APPlication OF TheORETICAL BACKGROUND. 4.1. Methodology to obtain sub-system's correlation function 4.1.1. From truth table to correlation function}

Let us take the "Business Strengths evaluation phase" presented at [1] and obtain its mathematical model. First, we'll need one bit to encode the "yes" and "no" supplier's qualifications; let us assign " 1 " and " 0 ", respectively. The resulting truth table is shown in Table 2.Additionally, let us apply the SOP technique. In doing so, we'll obtain the product terms shown in the last column. (Note: the symbol "l" indicates binary complement or "NOT" monary logic operator).

Table 2. Truth Table for the Business Strengths phase.

\begin{tabular}{|l|c|c|c|c|c|c|c|c|}
\hline $\begin{array}{l}\text { Criteria Decision } \\
\text { Pattern Number }\end{array}$ & X3 & X4 & X5 & X6 & X7 & $\begin{array}{c}\text { Output } \\
\text { Y2 }\end{array}$ & Product Term \\
\hline 1 & 0 & 0 & 0 & 0 & 0 & 0 & & \\
\hline$\ldots$ & 0 & X & X & X & X & 0 & & \\
\hline 16 & 0 & 1 & 1 & 1 & 1 & 0 & & \\
\hline 17 & 1 & 0 & 0 & 0 & 0 & 0 & & \\
\hline 18 & 1 & 0 & 0 & 0 & 1 & 0 & & \\
\hline 19 & 1 & 0 & 0 & 1 & 0 & 0 & \\
\hline 20 & 1 & 0 & 0 & 1 & 1 & 1 & X3.X4I.X51.X6.X7 \\
\hline 21 & 1 & 0 & 1 & 0 & 0 & $X$ & \\
\hline 22 & 1 & 0 & 1 & 0 & 1 & $X$ & & \\
\hline 23 & 1 & 0 & 1 & 1 & 0 & $X$ & & \\
\hline 24 & 1 & 0 & 1 & 1 & 1 & $X(1)$ & X3.X4I.X5.X6.X7 \\
\hline 25 & 1 & 1 & 0 & 0 & 0 & 0 & & \\
\hline 26 & 1 & 1 & 0 & 0 & 1 & 1 & X3.X4.X5I.X61.X7 \\
\hline 27 & 1 & 1 & 0 & 1 & 0 & 1 & & X3.X4.X5I.X6.X7। \\
\hline 28 & 1 & 1 & 0 & 1 & 1 & 1 & & X3.X4.X5I.X6.X7 \\
\hline 29 & 1 & 1 & 1 & 0 & 0 & 0 & & \\
\hline 30 & 1 & 1 & 1 & 0 & 1 & 1 & & X3.X4.X5.X61.X7 \\
\hline 31 & 1 & 1 & 1 & 1 & 0 & 1 & & X3.X4.X5.X6.X7। \\
\hline $32=\left(2^{5}\right)$ & 1 & 1 & 1 & 1 & 1 & 1 & X3.X4.X5.X6.X7 \\
\hline
\end{tabular}

Thus, considering all rows with " 1 " as output, the initial sub-system mathematical model would be:

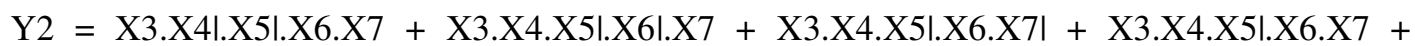
$\mathrm{X} 3 . \mathrm{X} 4 . \mathrm{X} 5 . \mathrm{X} 61 . \mathrm{X} 7+\mathrm{X} 3 . \mathrm{X} 4 . \mathrm{X} 5 . \mathrm{X} 6 . \mathrm{X} 7 \mathrm{I}+\mathrm{X} 3 . \mathrm{X} 4 . \mathrm{X} 5 . \mathrm{X} 6 . \mathrm{X} 7$

The symbol "X" on output means that the value could be either "0" or " 1 ", as is impossible that a supplier has 3 last financial years of business positive turnover (profit), while no 1 or 2 years. We'll decide about this during the minimization process, as will be shown forward.

\subsubsection{Finding the minimum expression with Boolean Algebra Axioms}

In order to find the minimal expression of Y2, the following axioms of Boolean algebra can be applied, being $\mathrm{X}, \mathrm{Y}$ and $\mathrm{Z}$, Boolean variables. 


$\begin{array}{rlc}\text { i. } & \mathbf{X} . \mathbf{Y}=\mathbf{Y} . \mathbf{X} & \text { (commutativity) } \\ \text { ii. } & \mathbf{1 . X}=\mathbf{X} & \text { (identity, the neutral on the product) } \\ \text { iii. } & \mathbf{X}+\mathbf{X}=\mathbf{X} & \text { (idempotency) } \\ \text { iv. } & \mathbf{X}+\mathbf{X} \mid=\mathbf{1} & \text { (complementarity) } \\ \text { v. } & \mathbf{X}+(\mathbf{Y}+\mathbf{Z})=(\mathbf{X}+\mathbf{Y})+\mathbf{Z}) & \text { (associativity) } \\ \text { vi. } & \mathbf{X} .(\mathbf{Y}+\mathbf{Z})=(\mathbf{X} . \mathbf{Y})+(\mathbf{X} . \mathbf{Z}) & \text { (distributivity) }\end{array}$

Thus, Y2can be rewritten as:

$\mathrm{Y} 2=(\mathrm{X} 3 \cdot \mathrm{X} 4 \cdot \mathrm{X} 5 \mathrm{I} \cdot \mathrm{X} 61 \cdot \mathrm{X} 7+\mathrm{X} 3 \cdot \mathrm{X} 4 \cdot \mathrm{X} 5 \mathrm{I} \cdot \mathrm{X} 6 \cdot \mathrm{X} 7+\mathrm{X} 3 \cdot \mathrm{X} 4 \cdot \mathrm{X} 5 \cdot \mathrm{X} 6 \mathrm{I} \cdot \mathrm{X} 7+\mathrm{X} 3 \cdot \mathrm{X} 4 \cdot \mathrm{X} 5 \cdot \mathrm{X} 6 \cdot \mathrm{X} 7)+$ (X3.X4.X51.X6.X7I + X3.X4.X5.X6.X7I + X3.X4.X51.X6.X7 + X3.X4.X5.X6.X7) + (X3.X4I.X5I.X6.X7 + X3.X4.X51.X6.X7 + X3.X4I.X5.X6.X7+X3.X4.X5.X6.X7)

Term in red colour corresponds to " $X$ " term. Looking at Table 2, the product terms considered were: $(26,28,30,32)+(27,31,28,32)+(20,28,24,32)$. The term 28 and 32 can be reused because of axiom iii.

Applying axioms $i, v i, i v$ and $i i$ :

$\mathrm{Y} 2=(\mathrm{X} 3 \cdot \mathrm{X} 4 \cdot \mathrm{X} 5 \mathrm{I} \cdot \mathrm{X} 7 \cdot(\mathrm{X} 61+\mathrm{X} 6)+\mathrm{X} 3 \cdot \mathrm{X} 4 \cdot \mathrm{X} 5 \cdot \mathrm{X} 7 \cdot(\mathrm{X} 61+\mathrm{X} 6))+$

$(\mathrm{X} 3 \cdot \mathrm{X} 4 \cdot \mathrm{X} 6 \cdot \mathrm{X} 7 \mathrm{l} \cdot(\mathrm{X} 5 \mathrm{I}+\mathrm{X} 5)+\mathrm{X} 3 \cdot \mathrm{X} 4 \cdot \mathrm{X} 6 \cdot \mathrm{X} 7 \cdot(\mathrm{X} 51+\mathrm{X} 5))+$

(X3. X5I.X6.X7.(X4I. + X4) + X3.X5.X6.X7.(X4I.+X4))

$=(\mathrm{X} 3 \cdot \mathrm{X} 4 \cdot \mathrm{X} 5 \mathrm{I} \cdot \mathrm{X} 7+\mathrm{X} 3 \cdot \mathrm{X} 4 \cdot \mathrm{X} 5 \cdot \mathrm{X} 7)+$

$(\mathrm{X} 3 . \mathrm{X} 4 . \mathrm{X} 6 . \mathrm{X} 7 \mathrm{I}+\mathrm{X} 3 . \mathrm{X} 4 . \mathrm{X} 6 . \mathrm{X} 7)+$

(X3.X51.X6.X7+X3.X5.X6.X7)

Then, $\mathrm{Y} 2$ can be finally minimized:

$\mathrm{Y} 2=\mathrm{X} 3 \cdot \mathrm{X} 4 \cdot \mathrm{X} 7+\mathrm{X} 3 \cdot \mathrm{X} 4 \cdot \mathrm{X} 6+\mathrm{X} 3 \cdot \mathrm{X} 6 \cdot \mathrm{X} 7$

If, as in [1], $\mathrm{X} 3$ should be " 1 ", Y2 can even reach the following form:

$\mathrm{Y} 2=\mathrm{X} 4 . \mathrm{X} 7+\mathrm{X} 4 . \mathrm{X} 6+\mathrm{X} 6 . \mathrm{X} 7$

\subsubsection{Finding the minimum expression with Karnaugh maps}

If we apply, as another alternative, Karnaugh maps, the resulting one will look as shown in Figure 1:

\begin{tabular}{|l|l|l|l|l|l|l|l|l|}
\hline X6X7 X3X4X5 & 000 & 001 & 011 & 010 & 110 & 111 & 101 & 100 \\
\hline 00 & & & & & & & & \\
\hline 01 & & & & & & & $\mathrm{X}$ & \\
\hline 11 & & & & & $\vdots$ & X & \\
\hline 10 & & & & & & & \\
\hline
\end{tabular}

Figure 1. Karnaugh Map the Business Strengths phase.

Groups of a $2^{n}$ number of " 1 's" should be identified, each of them generating a term in the output function; the larger the group the minimum of variables in the term. In this case, the three 4 ones 
size possible groups are: 1) cells in orange, generating the term:X3.X6.X7, which contains the variables who don't change, disappearing X3,X4,X5 who do change; 2) cells in horizontal lines, generating the term: X3.X4.X7; and 3) cells in vertical lines, generating the term: X3.X4.X6.

Hence, $\mathrm{Y} 2=\mathrm{X} 3 . \mathrm{X} 4 . \mathrm{X} 7+\mathrm{X} 3 . \mathrm{X} 4 . \mathrm{X} 6+\mathrm{X} 3 . \mathrm{X} 6 . \mathrm{X} 7$, the same obtained with Boolean algebra axioms.

In Karnaugh maps, any combination of the values of all input variables placed on any physical adjacent cell will have only a bit change between them. For example, first row-first column (000000) is adjacent with first row-second column (00100). Moreover, first row-first-column (00000), even not physically adjacent, will be logically with first row-eighth column (10000).

As can be seen in the example, " 1 's" can be reused, due to the fact that with Karnaugh maps we are implicitly and graphically applying the Boolean algebra axioms.

Therefore, the three groups in Figure 1 are of adjacent one's, eliminating as:

$$
n=\frac{\log x}{\log 2}
$$

variables associated with each group. In this case, all groups were 8 bits long, and so, two variables were eliminated from each term.

To demonstrate that this function performs identically with the version on [1], we create the Excel sheet shown in Figure 2.

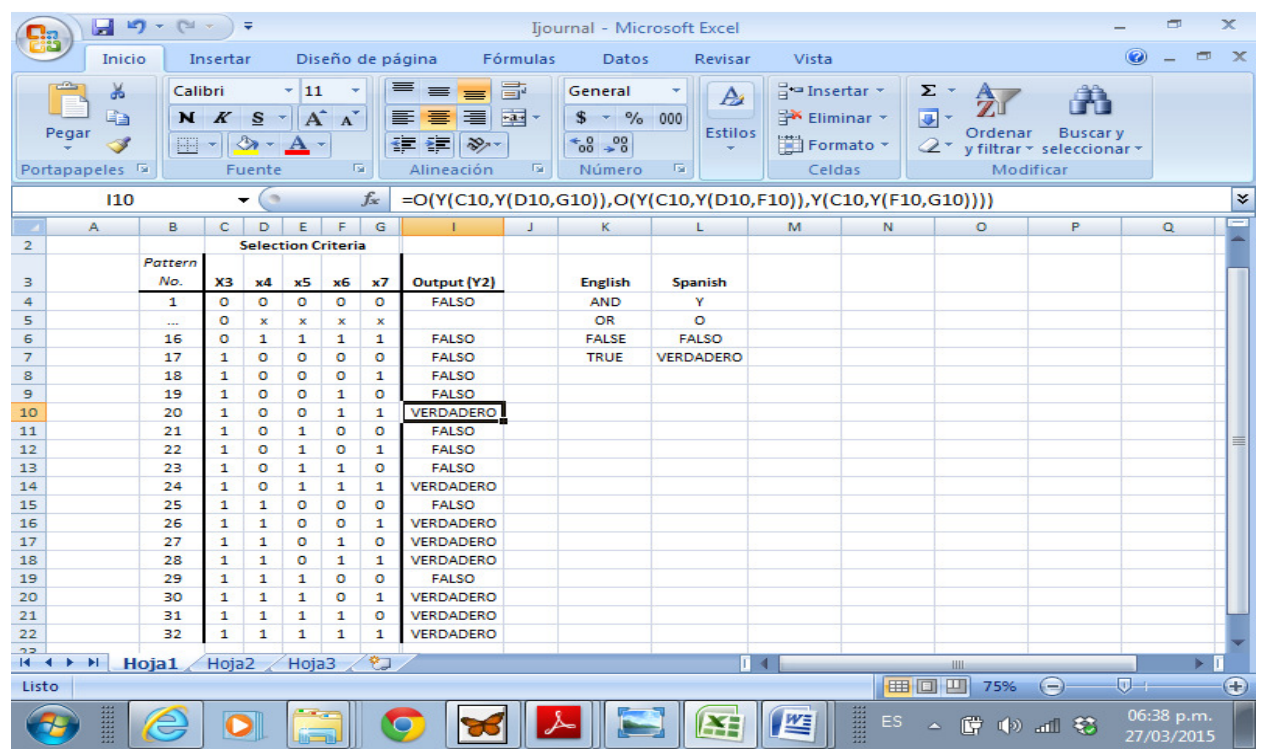

Figure 2. Karnaugh Map the Business Strengths phase.

\section{RESULTS}

\subsection{More than "yes" or "no" criteria qualification}

Let us take now the case of "Financial Quotation evaluation phase" in [1] to illustrate how this capacity is provided. Let's allow the Development (X9) criteria to be evaluated with four 
different degrees: excellent, good, average and poor. We'll code these with "11","10","01" and "00" 2-bit words, respectively. Besides, let's consider the policy to approve the suppliers qualified as "excellent" at "Development" (X9) criterion or those with at least an"average" note in combination with any other of the remaining criteria, X8 and X10. Thus, the resulting truth table will be as shown in Table 3.

Table 3. Truth Table for Technical phase.

\begin{tabular}{|c|c|c|c|c|c|}
\hline \multirow{2}{*}{$\begin{array}{l}\text { Decision Criteria } \\
\text { Pattern Number }\end{array}$} & \multirow[t]{2}{*}{ X8 } & \multicolumn{2}{|c|}{ X9 } & \multirow[t]{2}{*}{$\mathrm{X10}$} & \multirow{2}{*}{$\begin{array}{l}\text { Outpu1 } \\
\text { Y4' }\end{array}$} \\
\hline & & X9a & X9b & & \\
\hline 1 & 0 & 0 & 0 & 0 & 0 \\
\hline 2 & 0 & 0 & 0 & 1 & 0 \\
\hline 3 & 0 & 0 & 1 & 0 & 0 \\
\hline 4 & 0 & 0 & 1 & 1 & 1 \\
\hline 5 & 0 & 1 & 0 & 0 & 0 \\
\hline 6 & 0 & 1 & 0 & 1 & 1 \\
\hline 7 & 0 & 1 & 1 & 0 & 1 \\
\hline 8 & 0 & 1 & 1 & 1 & 1 \\
\hline 9 & 1 & 0 & 0 & 0 & 0 \\
\hline 10 & 1 & 0 & 0 & 1 & 0 \\
\hline 11 & 1 & 0 & 1 & 0 & 1 \\
\hline 12 & 1 & 0 & 1 & 1 & 1 \\
\hline 13 & 1 & 1 & 0 & 0 & 1 \\
\hline 14 & 1 & 1 & 0 & 1 & 1 \\
\hline 15 & 1 & 1 & 1 & 0 & 1 \\
\hline $16=\left(2^{4}\right)$ & 1 & 1 & 1 & 1 & 1 \\
\hline
\end{tabular}

The corresponding Karnaugh map is shown in Figure 3.

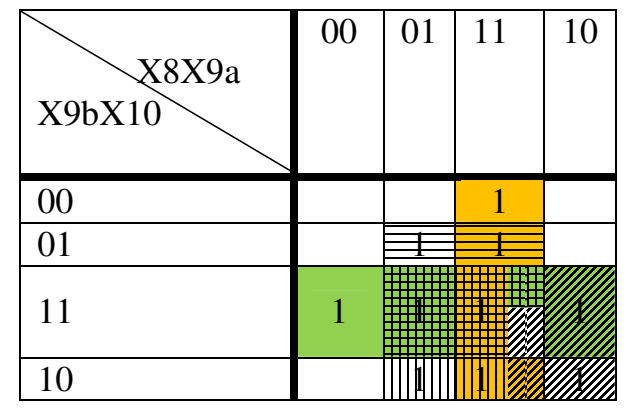

Figure 3. Karnaugh Map the Technical phase.

There are five groups of 4 one's, resulting in the following mathematical model:

$Y^{\prime}{ }^{\prime}=X 8 \cdot X 9 a+X 9 a \cdot X 10+X 9 b \cdot X 10+X 9 a \cdot X 9 b+X 8 \cdot X 9 b$

We can rewrite this on the following form:

$Y^{\prime}{ }^{\prime}=X 8 \cdot(X 9 a+X 9 b)+X 10 \cdot(X 9 a+X 9 b)+X 9 a \cdot X 9 b$

That will be easier to code as an Excel formula. 
This correlation function can be introduced to the Excel spreadsheet and incorporated to the whole automated decision support system for getting the decision-result for this entity, at this phase.

\section{CONCLUSIONS}

With our proposal, the BDM method has been provided with the capacity to qualify suppliers in a wider way, with the provision of the mechanism to operate.

Future works can revise the Quine-Mc Cluskey numeric method to obtain sub-systems mathematical model or other methods that could be found in the literature or recently developed. Also, good opportunities are in the application of this framework at specific scenarios.

\section{REFERENCES}

[1] N. Jigeesh, (2012) "A New Decision Support System for Supplier Selection using Boolean Algebra”, International Journal of Managing Public Sector Information and Communication Technologies, Vol. 5, No. 3, pp. 11-24.

[2] P. Scherz, (2000) "Practical electronics for inventors", New York: McGraw-Hill.

[3] R. Arnau,( 2015) "The application of Boolean algebra to industrial engineering decision problems", Master in Science in Industrial Engineering, Texas Technological College.

\section{Authors}

Gabriel Almazán, M. in Sc. (Telecommunications Engineering), Master (Educational Technology), working as a Full Time Lecturer and Researcher at Autonomous University of Hidalgo, México. Some of the topics he teaches are Industrial and Digital Electronics. His research interests include Business Intelligence, Sustainability, Industrial Symbiosis, Educational Technology, revitalization of native Mexican languages andself, cooperative and

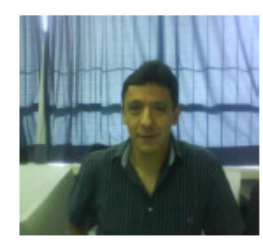
collaborative learning. 